\title{
Adjuvant corneal crosslinking to prevent hyperopic LASIK regression
}

This article was published in the following Dove Press journal:

Clinical Ophthalmology

28 March 2013

Number of times this article has been viewed

\section{loannis M Aslanides \\ Achyut N Mukherjee}

Emmetropia Mediterranean Eye Clinic, Heraklion, Crete, Greece
Correspondence: loannis M Aslanides Emmetropia Mediterranean Eye Clinic, Elephteria Square 44, Heraklion, Crete, 71201, Greece

$\mathrm{Tel}+302810226198$

Fax +30 28I 0343436

Email i.aslanides@emmetropia.gr
Purpose: To report the long term outcomes, safety, stability, and efficacy in a pilot series of simultaneous hyperopic laser assisted in situ keratomileusis (LASIK) and corneal crosslinking (CXL).

Method: A small cohort series of five eyes, with clinically suboptimal topography and/or thickness, underwent LASIK surgery with immediate riboflavin application under the flap, followed by UV light irradiation. Postoperative assessment was performed at 1, 3, 6, and 12 months, with late follow up at 4 years, and results were compared with a matched cohort that received LASIK only.

Results: The average age of the LASIK-CXL group was 39 years (26-46), and the average spherical equivalent hyperopic refractive error was +3.45 diopters (standard deviation 0.76 ; range 2.5 to 4.5 ). All eyes maintained refractive stability over the 4 years. There were no complications related to $\mathrm{CXL}$, and topographic and clinical outcomes were as expected for standard LASIK.

Conclusion: This limited series suggests that simultaneous LASIK and CXL for hyperopia is safe. Outcomes of the small cohort suggest that this technique may be promising for ameliorating hyperopic regression, presumed to be biomechanical in origin, and may also address ectasia risk.

Keyword: CXL

\section{Introduction}

Laser in situ keratomileusis (LASIK) is well established as the most commonly performed refractive surgical procedure. Despite incremental advances in technique and technology, LASIK still has some limitations with regard to risk and visual outcomes. One of the most serious complications of LASIK is post operative ectasia, where progressive corneal thinning is accompanied by refractive error and visual decline. ${ }^{1-3}$ LASIK is also limited with respect to refractive outcomes, due to inaccuracy of the treatment or, more commonly, subsequent regression. Both of these issues are more common in the treatment of higher ametropia, while regression is much more marked in hyperopic treatments. ${ }^{4}$

In recent years, the technique of corneal collagen crosslinking (CXL) has been introduced into clinical practice and is now widely used in the management of ectasia, and has been applied to the management of post-LASIK ectasia. ${ }^{5,6}$ In brief, CXL involves the application of riboflavin (vitamin B2) to the cornea and photoactivation by ultraviolet light, to allow the generation of reactive oxygen species. These effect a photochemical process involving a polymerization reaction of the corneal stromal 
collagen, which in turn, alters the biomechanical properties of the cornea, making it stiffer.,

The combination of CXL therapy with the LASIK procedure has the theoretical potential of ameliorating regression. Since corneal biomechanical response has been implicated in the pathophysiology of regression following hyperopic LASIK, modifying the corneal biomechanical properties might be beneficial. ${ }^{8,9}$ Regression is a considerable clinical problem warranting retreatment in a significant proportion of cases and restricting the effective range of treatment. The concurrent use of CXL with LASIK may also allow treatment of a broader range of cases at risk of ectasia from LASIK alone..$^{10,11}$

In this pilot study, we evaluated the long-term safety and stability of the CXL technique when used simultaneously with hyperopic LASIK. The main aims of this study were: (i) to identify any additional risks or complications induced by CXL, particularly over a longer term; (ii) to exclude any obvious effect of CXL on refractive outcome in hyperopic LASIK; and (iii) to evaluate the possibility that the treatment modifies the occurrence of regression. An early cohort of patients were thus prospectively followed up at 6 months and at 1,2 , and 4 years following treatment.

\section{Materials and methods}

The study patients were all recruited from a single unit (Emmetropia Mediterranean Eye Institute, Heraklion, Crete) from March to December of 2008. The study was approved by the Institute's ethics and research committee and fulfilled the principles of the Declaration of Helsinki. Informed consent was received from all patients. In this study, five eyes undergoing hyperopic LASIK were prospectively recruited and compared with a matched retrospective control group of five eyes undergoing LASIK only.

Inclusion criteria were age over 18 years and stable refraction for 12 months preceding surgery. Patients were only included where the preoperative assessment raised the possibility of suboptimal outcome, based on a clinical composite determination of corneal thickness, topography and magnitude of refraction. Four out of five eyes in the LASIKCXL group were assessed as high risk for ectasia, using the Randleman scoring system, while all control eyes were low risk. Three eyes, with corneal pathology, inflammation, glaucoma, and posterior segment pathology, were excluded.

All patients received treatment by a single surgeon (IM). The preoperative assessment included uncorrected distance visual acuity (UCDVA) and best corrected distance visual acuity (BCDVA) measurement, manifest and cycloplegic refraction, ultrasound pachymetry, corneal topography evaluation with Orbscan ${ }^{\circledR}$ (Bausch and Lomb Inc, Rochester, NY, USA) and Keratron Scout (Optikon ${ }^{\mathrm{TM}}$, Rome, Italy) topography systems. Slit-lamp exam and dilated fundus exam were performed on all subjects. All patients underwent standard aspheric treatment using the Amaris ${ }^{\circledR} 500 \mathrm{~Hz}$ flying spot excimer laser (SCHWIND, Kleinostheim, Germany).

Patients' eyes were anesthetized with proparacaine hydrochloride $0.5 \%$ (Alcaine ${ }^{\circledR}$; Alcon, Fort Worth, TX, USA). For the LASIK procedure, a MORIA M2 microkeratome (MORIA, Antony, France), with a 90 um head at speed 1(which cuts deeper flaps), was used to create a corneal flap. The stromal bed was dried prior to the excimer laser ablation. For the LASIK-CXL group, immediately after ablation, one drop of $0.1 \%$ riboflavin solution was instilled onto the bare stroma (Figure 1). The drop was left for 2 minutes and then was rinsed with balanced salt solution. The flap was then stretched back into place, and another riboflavin drop was instilled every 5 minutes alternating with a drop of balanced salt solution every 5 minutes. Finally, the flap was checked for striae and the symmetry of peripheral gutters. On completion of the LASIK procedure, CXL was carried out immediately, using an IROC UV-X ${ }^{\mathrm{TM}} 1000$ (IROC Innocross AG, Zug Switzerland) with an illumination of $3 \mathrm{~mW} / \mathrm{cm}^{2}$, for 30 minutes.

Postoperative assessments were performed on day 3 ; at 1 week; at 1, 3, and 6 months; and at 1, 2, 3, and 4 years (ending at 3 years in the control group), subject to attendance. During the follow-up visits UCDVA, BCDVA, and refraction were recorded, and slit lamp examination was performed. Statistical analysis was precluded based on the small series.

\section{Results \\ Demographics}

The pilot case series comprised five eyes from three hyperopic patients (LASIK-CXL group). Extended follow up was completed in 4 years in four of the eyes and in 3 years in one eye.

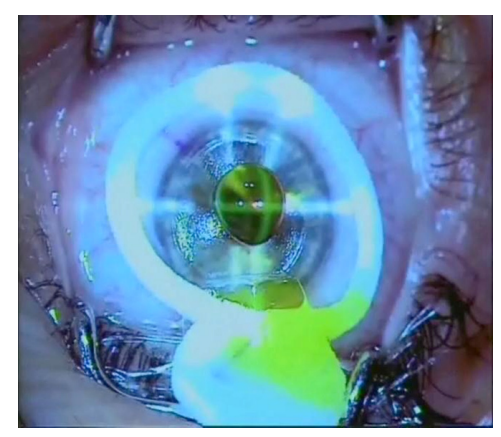

Figure I Application of riboflavin to the stromal bed immediately following hyperopic LASIK.

Abbreviation: LASIK, laser assisted in situ keratomileusis. 
Table I Corneal crosslinking for hyperopic lasik: individual case data, mean and control group mean values

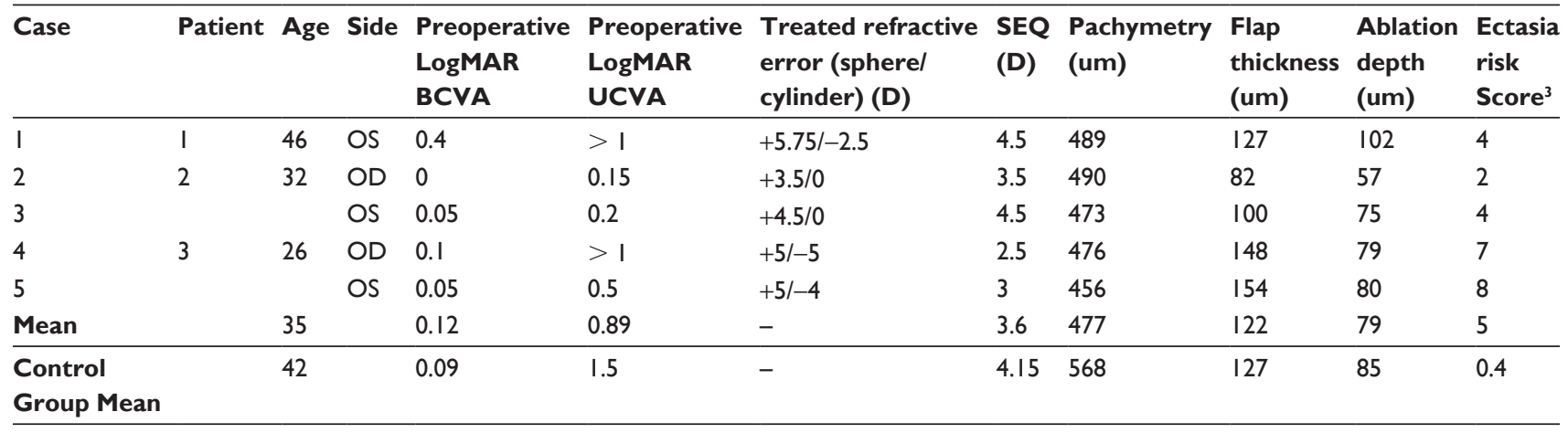

Abbreviations: UCVA, Uncorrected Visual Acuity; BCVA, Best Corrected Visual Acuity; SEQ, Spherical Equivalent.

A matched control group of five eyes from five patients was selected that was close to the treated refractive error, from patients undergoing standard LASIK only. Follow up was available for 3 years in the controls. The average age was 39 years (range 26 to 46 ) in the LASIK-CXL group and 42 years (28 to 49 ) in the control group. Mean hyperopic spherical equivalent refractive error was 3.6 diopters (D) (standard deviation [SD] 2.1; range 2.5 to 4.5 ) in the LASIK-CXL group and 4.15 D (SD 2.0; range 1.5 to 6.25 ) in the control group. The mean astigmatic error was 2.3 $\mathrm{D}$ (SD 2.3; range 0 to 5) in the LASIK-CXL group and 1.6 (SD 1.2; range 0 to 3.25) in the control group. The average planned stromal ablation was $78.6 \mu \mathrm{m}$ (SD 15.8; range 57.5 to 101.9 ) in the LASIK and $85 \mu \mathrm{m}$ (SD 30.1; range 50 to 115 ) in the controls. The average flap depth, measured intraoperatively by subtraction ultrasound pachymetry, was $122.2 \mu \mathrm{m}$ (SD 30.8; range 82 to 154 ) in the LASIK-CXL group and $127.4 \mu \mathrm{m}$ (SD 23.0, range 100 to 156 ) in the control group. Individual case data, means, and control group means are presented in Table 1 .

There were no perioperative complications. Careful slitlamp biomicroscopy was carried out to exclude flap-related complications, such as striae or dislocation. Following the CXL treatment, all flaps behaved clinically, as for routine LASIK. During the early postoperative period, no delay of reepithelization was noted. A faint midstromal haze attributed to CXL was noted from the first postoperative day but had mostly resolved by 1 week follow up. There was no discernible residual haze from 1 month onwards.

\section{Outcomes}

The mean preoperative LogMAR equivalent BCVA was 0.12 and UDVA was 0.89 , for the LASIK-CXL group; BCVA was 0.09 and UDVA was 1.5 , in the controls. One eye in the LASIK-CXL group had a BCVA of 0.4 LogMAR due to amblyopia. Mean follow up was 48 months (37 to 55) in the LASIK-CXL group, which underwent extended follow up.
The control group was retrospective, with all cases followed up to 36 months. At final follow up, postoperative mean LogMAR UDVA and BCVA were 0.09 and 0.017 , respectively, in the LASIK-CXL group, and UDVA and BCVA were 0.17 and 0.06 , respectively, in the control group. In terms of safety, no eye lost lines of best corrected vision. Two eyes gained a line of best corrected vision in the LASIK-CXL group, and there was no significant change of two lines or more in either group. The accuracy of refractive correction in the LASIK-CXL group appeared predictable, with $4(80 \%)$ eyes achieving a zero spherical equivalent postoperative refraction and one eye having myopia of $0.25 \mathrm{D}$ at 1 month follow up. The accuracy of intended vs achieved refractive outcomes in the LASIKCXL group are shown in Figure 2. Astigmatism was reduced from mean 2.3 D (SD 2.3) preoperatively to $0.3 \mathrm{D}(\mathrm{SD} 0.4)$ at 1 month in the LASIK-CXL group and from 1.6 D (SD 1.2) to $0.25 \mathrm{D}$ (SD 0.3) in the LASIK-only group. All eyes in the LASIK-CXL group showed refractive stability through the extended follow up, with no apparent hyperopic regression,

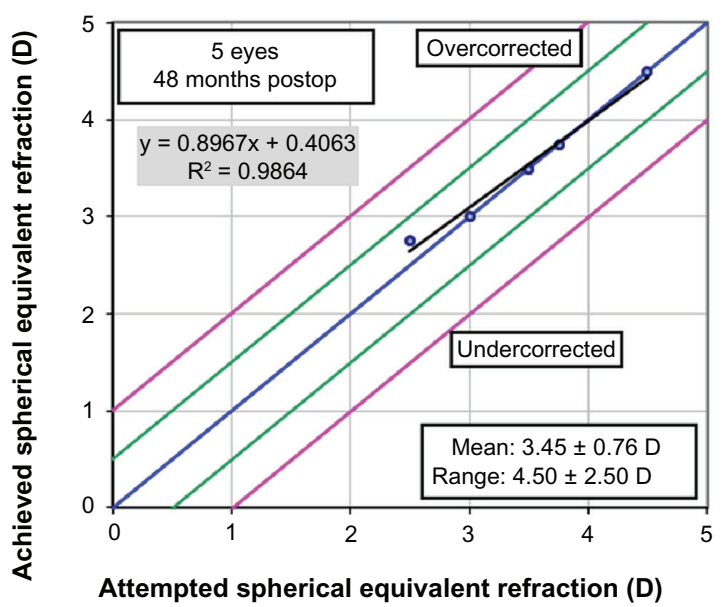

Figure 2 Attempted vs achieved refractive outcomes in hyperopic LASIK with simultaneous corneal crosslinking.

Abbreviations: LASIK, laser assisted in situ keratomileusis; D, diopters. 

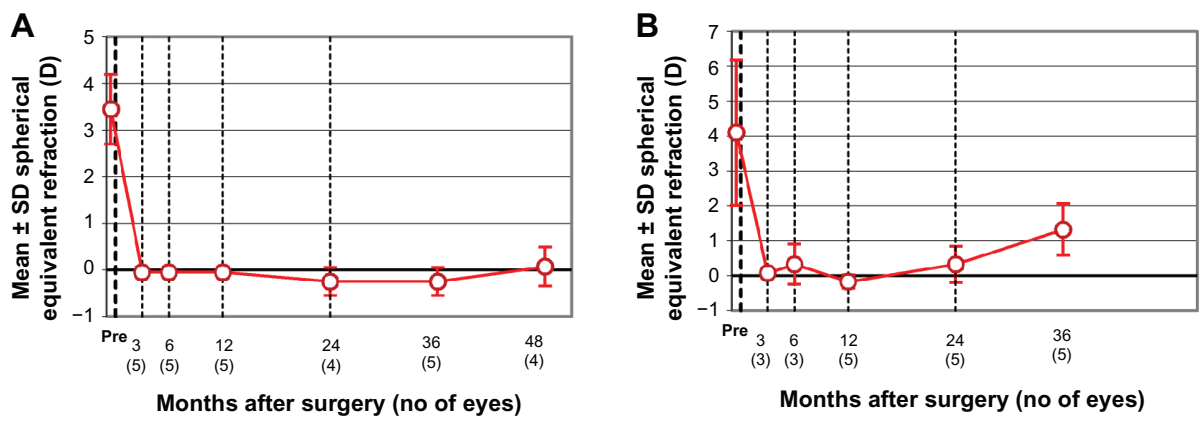

Figure 3 Stability of refractive correction in (A) hyperopic LASIK with simultaneous corneal crosslinking (LASIK-CXL); and (B) a matched control group. Abbreviations: LASIK, laser assisted in situ keratomileusis; CXL, crosslinking; SD, standard deviation; D, diopter.

while in the LASIK-only group, there was a suggestive trend towards hyperopic regression (Figure 3).

\section{Discussion}

The primary finding of this pilot study was that the addition of simultaneous CXL therapy to hyperopic LASIK does not appear to have any obvious deleterious effect on the safety of the procedure, even after long-term follow up. Specifically, we did not observe any clinical or topographic evidence of flaprelated complications or late induced changes. There was some early stromal haze noted, but this did not seem to significantly limit visual recovery and resolved within a month. Our study indicates that UCDVA quickly reaches the same levels as after a standalone LASIK procedure, without inducing any side effects or compromising visual safety. Hafezi and Kanellopoulos et $\mathrm{al}^{6}$ introduced the use of CXL for LASIK-induced ectasia and adjunctive higher-fluence CXL during LASIK. Subsequent series of accelerated CXL for myopic LASIK have had similar safety findings at short term follow up. ${ }^{6,10,11}$

A secondary consideration is the effect of CXL therapy on the laser nomogram. Kampik et al, ${ }^{12}$ in animal studies, demonstrated a reduced treatment effect for myopic LASIK in CXL corneas, although ablation rates were unaffected, suggesting altered biomechanical response. However, we found no dramatic pattern of over- or undercorrection attributable to the combination of CXL and hyperopic treatment. There was also no suggestion of increased variability. The possibility of a slightly altered treatment nomogram cannot be addressed by this small series. Two recent clinical series assessing myopic LASIK with concurrent CXL similarly found no effect on refractive correction. ${ }^{10,11}$

The primary goal of CXL hyperopic LASIK is to minimize regression. This series is insufficient to provide a conclusion in this regard, but it is notable that there was no significant hyperopic regression at 4 years follow up, for any eye. This is more notable given that these were eyes with higher hyperopia and astigmatism, with relatively thin preoperative pachymetry, and with high-risk ectasia scores, which increased their risk of biomechanical changes. ${ }^{9} \mathrm{CXL}$ may alter the response to LASIK in a number of ways. Mi et $\mathrm{l}^{13}$ found, in an organ culture model, that apart from the change in biomechanical rigidity of the residual stroma, the adhesion of the flap itself was doubled by CXL, which may have additional benefits to regression.

To our knowledge, no previous peer-reviewed study has evaluated the usage of CXL simultaneously with hyperopic LASIK surgery. Additionally, no study has evaluated the longer-term risks of adding CXL to the established LASIK procedure. As no adverse effects were noted in this series, we believe further evaluation of the role of CXL in reducing regression following hyperopic LASIK to be warranted, in addition to evaluation of its previously suggested role in ameliorating ectasia risk.

\section{Disclosure}

The authors report no conflicts of interest in this work.

\section{References}

1. Pallikaris IG, Kymionis GD, Astyrakakis NI. Corneal ectasia induced by laser in situ keratomileusis. J Cataract Refract Surg. 2001;27(11):1796-1802.

2. Binder PS. Analysis of ectasia after laser in situ keratomileusis: risk factors. $J$ Cataract Refract Surg. 2007;33(9):1530-1538.

3. Randleman JB, Woodward M, Lynn MJ, Stulting RD. Risk assessment for ectasia after corneal refractive surgery. Ophthalmology. 2008;115(1):37-50.

4. Settas G, Settas C, Minos E, Yeung IY. Photorefractive keratectomy (PRK) versus laser assisted in situ keratomileusis (LASIK) for hyperopia correction. Cochrane Database Syst Rev. 2012;6:CD007112.

5. Wollensak G, Spoerl E, Seiler T. Riboflavin/ultraviolet-a-induced collagen crosslinking for the treatment of keratoconus. Am J Ophthalmol. 2003;135(5):620-627.

6. Hafezi F, Kanellopoulos J, Wiltfang R, Seiler T. Corneal collagen crosslinking with riboflavin and ultraviolet $\mathrm{A}$ to treat induced keratectasia after laser in situ keratomileusis. J Cataract Refract Surg. 2007;33(12):2035-2040.

7. Kolli S, Aslanides IM. Safety and efficacy of collagen crosslinking for the treatment of keratoconus. Expert Opin Drug Saf. 2010;9(6):949-957. 
8. de Medeiros FW, Sinha-Roy A, Alves MR, Wilson SE, Dupps WJ Jr. Differences in the early biomechanical effects of hyperopic and myopic laser in situ keratomileusis. J Cataract Refract Surg. 2010;36(6):947-953.

9. Dupps WJ Jr, Wilson SE. Biomechanics and wound healing in the cornea. Exp Eye Res. 2006;83(4):709-720.

10. Kanellopoulos AJ. Long-term safety and efficacy follow-up of prophylactic higher fluence collagen cross-linking in high myopic laserassisted in situ keratomileusis. Clin Ophthalmol. 2012;6:1125-1130.

11. Celik HU, Alagöz N, Yildirim Y, et al. Accelerated corneal crosslinking concurrent with laser in situ keratomileusis. J Cataract Refract Surg. 2012;38(8):1424-1431.
12. Kampik D, Ralla B, Keller S, Hirschberg M, Friedl P, Geerling G. Influence of corneal collagen crosslinking with riboflavin and ultraviolet-a irradiation on excimer laser surgery. Invest Ophthalmol Vis Sci. 2010;51(8):3929-3934.

13. Mi S, Dooley EP, Albon J, Boulton ME, Meek KM, Kamma-Lorger CS. Adhesion of laser in situ keratomileusis-like flaps in the cornea: Effects of crosslinking, stromal fibroblasts, and cytokine treatment. J Cataract Refract Surg. 2011;37(1):166-172.
Clinical Ophthalmology

\section{Publish your work in this journal}

Clinical Ophthalmology is an international, peer-reviewed journal covering all subspecialties within ophthalmology. Key topics include: Optometry; Visual science; Pharmacology and drug therapy in eye diseases; Basic Sciences; Primary and Secondary eye care; Patien Safety and Quality of Care Improvements. This journal is indexed on

Submit your manuscript here: http://www.dovepress.com/clinical-ophthalmology-journal

\section{Dovepress}

PubMed Central and CAS, and is the official journal of The Society of Clinical Ophthalmology (SCO). The manuscript management system is completely online and includes a very quick and fair peer-review system, which is all easy to use. Visit http://www.dovepress.com/ testimonials.php to read real quotes from published authors. 